\title{
Anomaly of spontaneous transition to instability of liquid-vapour front in a porous medium
}

\begin{abstract}
In this article, we have investigated the instability of the liquid-vapour front in a geothermal system with isothermal boundaries. A two-dimensional linear stability analysis of the isothermal basic state shows that the RayleighTaylor mechanism is the dominant contributor to instability. A conditional expression for the critical modified Rayleigh number for different heat transport processes has been found. It has been shown that the spontaneous transition to instability is an artefact of neglecting thermal advection and the imposition of the phase change front to be equidistant from the liquid and vapour boundaries.
\end{abstract}

Keywords: Geothermal system, liquid-vapour front, spontaneous transition, Rayleigh-Taylor instability

\section{Introduction}

In 1883 Lord Rayleigh described for the first time the instability of a dense fluid overlying a lower density fluid in a gravitational field, which is known as the Rayleigh-Taylor instability [1]. The transitions to instability at fluid-fluid interfaces are of great interest on account of their wide range of applications. These instabilities can often occur at a liquid-vapour interface in a geothermal system [2-10]. There is much need for the better understanding of the different physical phenomena involved with liquid-vapour phase changes, and this is the focus of our study.

The term "spontaneous" transition in continuum mechanics refers to a special case of instability; when all wave numbers become unstable at the same value of the controlling parameter. The "spontaneous" transition of Il'ichev \& Tsypkin $[11,12]$ is shown to be a very unusual case, depending not only on the front position but also on the neglect of advective heat transport. Tsypkin \& Il'ichev [11, 12] investigated different cases of transition 
to instability of a stationary vertical phase change flow under the condition that conduction dominates over advection. It was shown that if the interface is equidistant from the liquid and vapour boundaries then there is a spontaneous transition to instability. A spontaneous transition to instability is a very unusual phenomenon and so it is worth investigating more thoroughly the conditions under which it can occur. In particular, (i) is it an artefact of the relationship assumed by Tsypkin \& Il'ichev [11] between the phase-change temperature and the pressure, or does it occur in other models; and (ii) is it crucial that advective heat transport is neglected?

Tsypkin \& Il'ichev [7] using typical values for physical quantities, deduce that advection may be neglected if

$$
K\left|\delta P-\rho_{w} g L\right| \ll 10^{-10} \mathrm{~N},
$$

where $K$ is permeability, $\delta P$ is the pressure difference across the layer, $L$ is the characteristic length scale, $g$ is gravity and $\rho_{w}$ is the density of water. If we assume that $\left|\delta P-\rho_{w} g L\right|$ is of the same order of magnitude as $\rho_{w} g L$ (i.e. that the applied pressure difference is roughly comparable in magnitude to the hydrostatic pressure across the layer) then (1) simplifies to

$$
K \rho_{w} g L \ll 10^{-10} \mathrm{~N},
$$

or, using $\rho_{w}=1000 \mathrm{~kg} \mathrm{~m}^{-3}$ and $g \approx 10 \mathrm{~m} \mathrm{~s}^{-2}$, then (2) yields

$$
K L \ll 10^{-14} \mathrm{~m}^{3} \text {. }
$$

Thus for a sandstone with $\mathrm{K}=10^{-10} \mathrm{~m}^{2}$, advection can be neglected only if $L \ll 10^{-4} \mathrm{~m}$, i.e. for any large-scale aquifer advection is important. For a granitic rock with $K=10^{-16} \mathrm{~m}^{2}$, advection can be neglected only if $L \ll 10^{2}$ $\mathrm{m}$, so it may be negligible under these conditions.

In the present study, the basic vertical flow is without the phase motion through the interface. We employ a simpler relation of temperature and pressure at the liquid-vapour interface $\left(T=T_{S}(P)\right)$ by assuming a constant temperature and a continuous pressure at the front and a more complete heat transport equation. Both the pressure and the temperature profiles for the base flow are linearly distributed. The important aspect of this analysis is that we will consider a more realistic perturbed state accounting for thermal advection. The Il'ichev \& Tsypkin $[7,11]$ analysis will be studied as a special case. We will show that the transition to instability is not spontaneous as found by Il'ichev \& Tsypkin $[7,11]$, indicating that the interesting behaviour in their model is an artefact of taking a very simple model which neglected thermal advection. 


\section{Mathematical Model}

We consider a uniform, isotropic and fully fluid saturated porous layer of infinite extension bounded by two horizontal, much more permeable layers. The upper and lower highly permeable layers are filled either with vapour and liquid, respectively or liquid and vapour, respectively (see Fig. 1). In the low-permeability layer there exists a phase change front which separates the liquid phase from the vapour phase.

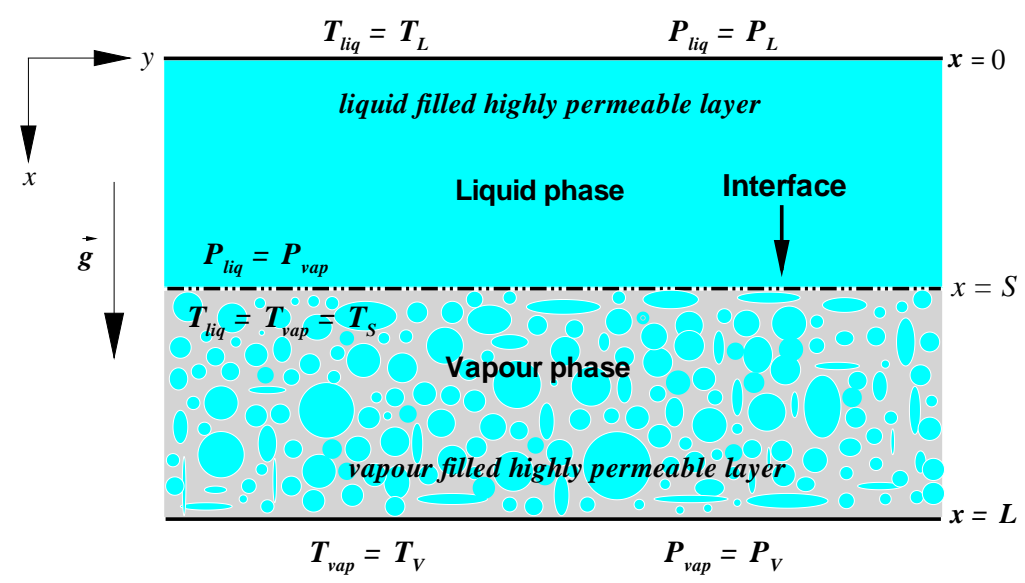

Figure 1: Schematic diagram of the proposed problem.

\subsection{Governing equations}

The continuity equation for incompressible flow in dimensionless form is

$$
\frac{\partial u_{l i q, v a p}^{*}}{\partial x^{*}}+\frac{\partial v_{l i q, v a p}^{*}}{\partial y^{*}}=0 .
$$

Darcy's equation is taken to hold in each phase and are presented in scaled form as

$$
\begin{array}{lll}
u_{l i q}^{*}=-\left(\frac{\partial P_{l i q}^{*}}{\partial x^{*}}+R_{3}\right), & u_{v a p}^{*}=-\frac{R_{1} R_{2} \kappa}{C}\left(\frac{\partial P_{v a p}^{*}}{\partial x^{*}}+R_{1} R_{3}\right), \\
v_{l i q}^{*}=-\frac{\partial P_{l i q}^{*}}{\partial y^{*}}, & v_{v a p}^{*}=-\frac{R_{1} R_{2} \kappa}{C} \frac{\partial P_{v a p}^{*}}{\partial y^{*}} .
\end{array}
$$

In the equations above, $R_{3}$ is the modified Rayleigh number which has been defined as

$$
R_{3}=\frac{K \rho_{l i q}^{2} c_{p_{l i q}} g L}{\mu_{l i q} k_{m, l i q}}
$$


where $K$ is the permeability of the homogeneous medium and $g$ is the acceleration due to gravity with $x^{*}$-co-ordinate increasing downwards. The modified Rayleigh number $R_{3}$ will be our key quantity for understanding the Rayleigh-Taylor instability in a geothermal system.

We will use the one-equation model to describe the heat transport in the porous medium assuming local thermal equilibrium. In dimensionless form, the equations in the liquid and vapour regions become

$$
\left.\begin{array}{l}
E_{l i q} \frac{\partial \Theta_{l i q}}{\partial t^{*}}+u_{l i q}^{*} \frac{\partial \Theta_{l i q}}{\partial x^{*}}+v_{l i q}^{*} \frac{\partial \Theta_{l i q}}{\partial y^{*}}=\frac{\partial^{2} \Theta_{l i q}}{\partial x^{* 2}}+\frac{\partial^{2} \Theta_{l i q}}{\partial y^{* 2}}, \\
\frac{E_{v a p} \kappa R_{1}}{C} \frac{\partial \Theta_{v a p}}{\partial t^{*}}+u_{v a p}^{*} \frac{\partial \Theta_{v a p}}{\partial x^{*}}+v_{v a p}^{*} \frac{\partial \Theta_{v a p}}{\partial y^{*}}=\frac{\partial^{2} \Theta_{v a p}}{\partial x^{* 2}}+\frac{\partial^{2} \Theta_{v a p}}{\partial y^{* 2}} .
\end{array}\right\}
$$

It can be seen from the above equations that the energy transport is coupled with the mass transport, which introduces non-linearities. But this is not the only reason for the inherited non-linearities. The other reason is the coupling of the interface position with the heat and mass transport equation.

The most important aspect of phase change problems is the energy and mass balance at the interface of the two phases, which makes the problem non-linear. The energy jump condition at the interface $x=S(y, t)$ is

$$
\begin{aligned}
\varphi H_{l i q} \frac{\partial S^{*}}{\partial t}=\left\{\frac{\partial \Theta_{l i q}}{\partial x^{*}}-\frac{\partial S^{*}}{\partial y^{*}} \frac{\partial \Theta_{l i q}}{\partial y^{*}}\right\}_{x^{*}=S^{*}}+\frac{\Theta_{0}}{\kappa} & \left\{\frac{\partial \Theta_{v a p}}{\partial x^{*}}-\frac{\partial S^{*}}{\partial y^{*}} \frac{\partial \Theta_{v a p}}{\partial y^{*}}\right\}_{x^{*}=S^{*}} \\
& -H_{l i q}\left(\frac{\partial P_{l i q}^{*}}{\partial x^{*}}+R_{3}\right)
\end{aligned}
$$

This energy jump condition shows that the liquid-vapour interface position depends on the temperature distributions in both phases as well as on the transfer of fluid across the front (evaporation or condensation).

Furthermore the mass jump condition at the interface is also coupled with the velocity profiles in both phases, giving in scaled form

$\varphi\left(1-R_{1}\right) \frac{\partial S^{*}}{\partial t^{*}}=\left.R_{1} R_{2} \frac{\partial P_{v a p}^{*}}{\partial x^{*}}\right|_{x^{*}=S^{*}}-\left.\frac{\partial P_{l i q}^{*}}{\partial x^{*}}\right|_{x^{*}=S^{*}}-R_{3}\left(1-R_{1}^{2} R_{2}\right)$. 
The governing equations and the boundary conditions are made dimensionless using the following reference parameters,

$$
\left.\begin{array}{l}
x^{*}=\frac{x}{L}, \quad S^{*}=\frac{S}{L}, \quad \kappa=\frac{k_{m, l i q}}{k_{m, v a p}}, \quad C=\frac{c_{p, l i q}}{c_{p, v a p}}, \quad \Theta_{l i q}\left(x^{*}\right)=\frac{T_{l i q}(x)-T_{L}}{T_{S}-T_{L}}, \\
\Theta_{v a p}\left(x^{*}\right)=\frac{T_{V}-T_{v a p}(x)}{T_{V}-T_{S}}, \quad \Theta_{0}=\frac{T_{V}-T_{S}}{T_{S}-T_{L}}, \quad P^{*}=\frac{K \rho_{l i q} c_{p l i q} P}{\mu_{l i q} k_{m, l i q}}, \\
u_{l i q, v a p}^{*}=\frac{L \rho_{l i q, v a p} c_{p_{l i q}, v a p} u_{l i q, v a p}}{k_{m, l i q, v a p}}, \quad v_{l i q, v a p}^{*}=\frac{L \rho_{l i q, v a p} c_{p l i q, v a p} v_{l i q, v a p}}{k_{m, l i q, v a p}}, \\
H_{l i q}=\frac{\lambda}{c_{p, l i q}\left(T_{S}-T_{L}\right)}, \quad R_{1}=\frac{\rho_{v a p}}{\rho_{m, l i q}}, \quad R_{2}=\frac{\mu_{l i q}}{\mu_{v a p}}, \quad E_{l i q}=\frac{\left(\rho c_{p}\right)_{m, l i q}}{\left(\rho c_{p}\right)_{l i q}}, \\
E_{v a p}=\frac{\left(\rho c_{p}\right)_{m, v a p}}{\left(\rho c_{p}\right)_{v a p}}, t=\frac{L^{2} \rho_{l i q} c_{p l i q} t^{*}}{k_{m, l i q}}
\end{array}\right\}
$$

where $H_{l i q}$ is the reciprocal of the Stefan number for the liquid region, and represents the ratio of the latent heat $\lambda$ to the sensible heat $\left(T_{S}-T_{L}\right)[13$, p. 91]. The pressure scale is based on these characteristic velocities and the resistance of the medium to liquid flow. The ratio of the temperature contrasts across the liquid and vapour layers is denoted by $\Theta_{0}$. The present model is the same as was considered in references [10, 14, 15]. For details of the notation, especially the non-dimensionalisation parameters, the reader is referred to these references.

\section{Linear Stability analysis}

In this section, we will discuss the stability of a steady liquid-vapour phase change front in a porous medium with isothermal boundaries and no through flow. To examine the stability of the liquid-vapour interface, an infinitesimal disturbance is applied to the basic state. The aim is to linearise the governing equations and boundary conditions about the basic state and to study the behaviour of the perturbed interface.

\subsection{Perturbed form of the problem}

The temperature, velocity, pressure field both in the liquid and vapour regions and the liquid-vapour phase change front are expanded in the following manner

$$
\left.\begin{array}{l}
\Theta_{l i q}=\Theta_{l i q}^{0}\left(x^{*}\right)+\epsilon \Theta_{l i q}^{1}\left(x^{*}, y^{*}, t^{*}\right), \quad \Theta_{v a p}=\Theta_{v a p}^{0}\left(x^{*}\right)+\epsilon \Theta_{v a p}^{1}\left(x^{*}, y^{*}, t^{*}\right), \\
u_{l i q}^{*}=\omega_{0}+\epsilon \omega_{1}, \quad u_{v a p}^{*}=\Omega_{0}+\epsilon \Omega_{1}, \quad v_{l i q}^{*}=\Gamma_{0}+\epsilon \Gamma_{1}, \quad v_{v a p}^{*}=\Upsilon_{0}+\epsilon \Upsilon_{1}, \\
P_{l i q}^{*}=\Lambda_{0}+\epsilon \Lambda_{1}, \quad P_{v a p}^{*}=\Pi_{0}+\epsilon \Pi_{1}, \quad S^{*}=S_{0}^{*}+\epsilon S_{1}^{*}\left(y^{*}, t^{*}\right),
\end{array}\right\}
$$

and only the first order terms in $\epsilon$ are retained [16, p. 48], where $0<$ $\epsilon \ll 1$. The small perturbation parameter $\epsilon$ represents the magnitude of 
the deviation from the basic state. The subscript and superscript 0 and 1 denote the steady state and perturbed state, respectively.

\subsection{Steady state}

The basic state of the system is assumed to be steady and independent of the horizontal variable $y^{*}$, so $\Lambda_{0}, \Theta_{\text {liq }}^{0}, \Pi_{0}$ and $\Theta_{v a p}^{0}$ are all functions of $x^{*}$ only. Also the fluid is stationary, $\omega_{0}=\Omega_{0}=\Gamma_{0}=\Upsilon_{0}=0$. The temperature profile is assumed to be conductive and the phase change front is static, so the equations governing the basic state will take the form

$$
\text { pressure profile }\left\{\begin{array}{l}
\frac{d \Lambda_{0}}{d x^{*}}=-R_{3}, \quad \Lambda_{0}(0)=P_{L}^{*}, \\
\frac{d \Pi_{0}}{d x^{*}}=-R_{1} R_{3}, \Lambda_{0}\left(S_{0}^{*}\right)=\Pi_{0}\left(S_{0}^{*}\right),
\end{array}\right.
$$

and

$$
\text { temperature profile }\left\{\begin{array}{l}
\frac{d^{2} \Theta_{l i q}^{0}}{d x^{* 2}}=0, \quad \Theta_{l i q}^{0}(0)=0, \Theta_{l i q}^{0}\left(S_{0}^{*}\right)=1, \\
\frac{d^{2} \Theta_{v a p}^{0}}{d x^{* 2}}=0, \quad \Theta_{v a p}^{0}(1)=0, \Theta_{v a p}^{0}\left(S_{0}^{*}\right)=1, \\
\frac{d \Theta_{l i q}^{0}}{d x^{*}}+\frac{\Theta_{0}}{\kappa} \frac{d \Theta_{v a p}^{0}}{d x^{*}}=0 .
\end{array}\right.
$$

The stationary solutions of (8) and (9) give the linear pressure profile and the purely conductive temperature profiles in the liquid and the vapour regions

$$
\left.\begin{array}{l}
\Lambda_{0}=P_{L}^{*}-R_{3} x^{*}, \quad \Pi_{0}=P_{L}^{*}+R_{3} S_{0}^{*}\left(R_{1}-1\right)-R_{1} R_{3} x^{*}, \\
\Theta_{\text {liq }}^{0}=\frac{x^{*}}{S_{0}^{*}}, \quad \Theta_{\text {vap }}^{0}=\frac{x^{*}-1}{S_{0}^{*}-1} .
\end{array}\right\}
$$

The front position in the steady state is

$$
S_{0}^{*}=\frac{\kappa}{\kappa+\Theta_{0}} .
$$

The above equation (11) shows that in the absence of net flow in the reservoir, the front position depends on the ratio of the temperature contrast $\left(\Theta_{0}\right)$ and the ratio of thermal conductivities $(\kappa)$ of the two phases. It will helpful to predict the different positions of the liquid-vapour interface, while assuming some special properties of these two controlling parameters; 
1. If $\kappa \rightarrow 0$ then $S_{0}^{*} \rightarrow 0$. (the system is dominated by the vapour phase)

2. If $\kappa=\Theta_{0}$ then $S_{0}^{*}=\frac{1}{2}$. (the interface is equidistant from the liquid and vapour boundaries)

3. If $\Theta_{0} \rightarrow 0$ then $S_{0}^{*} \rightarrow 1$. (the system is dominated by the vapour phase)

\subsection{The eigenvalue problem}

According to the classical procedure [16, p. 49], the pressure and temperature profiles in both regions (liquid and vapour) and the phase change interface location in the first order problem are expanded in normal modes,

$$
\left(\Theta_{l i q}^{1}, \Theta_{\text {vap }}^{1}, \Lambda_{1}, \Pi_{1}, S_{1}^{*}\right)=\left(\phi_{l i q}\left(x^{*}\right), \phi_{v a p}\left(x^{*}\right), \Psi\left(x^{*}\right), \Sigma\left(x^{*}\right), \Phi\right) \exp \left[\sigma t^{*}+i l y^{*}\right],
$$

where $\phi_{\text {liq }}, \phi_{v a p}$ and $\Phi$ are the eigenfunctions of temperature in the liquid region, temperature in the vapour region and the interface location, respectively, and $l$ and $\sigma$ denote the wave number and the rate of growth (or decay) of the disturbance. The eigenfunctions of pressure in both phases (liquid and vapour) are denoted by $\Psi$ and $\Sigma$, respectively.

The eigenvalue problem with the appropriate boundary conditions for the pressure profiles in both phases (liquid and vapour) is

$$
\left.\begin{array}{l}
\frac{d^{2} \Psi\left(x^{*}\right)}{d x^{* 2}}-l^{2} \Psi\left(x^{*}\right)=0, \quad \Psi(0)=0, \Sigma(1)=0, \\
\frac{d^{2} \Sigma\left(x^{*}\right)}{d x^{* 2}}-l^{2} \Sigma\left(x^{*}\right)=0, \Psi\left(S_{0}^{*}\right)=\Sigma\left(S_{0}^{*}\right)+\Phi R_{3}\left(1-R_{1}\right), \\
\varphi\left(1-R_{1}\right) \sigma \Phi=\left.R_{1} R_{2} \frac{\partial \Sigma}{\partial x^{*}}\right|_{x^{*}=S_{0}^{*}}-\left.\frac{\partial \Psi}{\partial x^{*}}\right|_{x^{*}=S_{0}^{*}} .
\end{array}\right\}
$$

The solutions of (13) are

$$
\text { pressure profile }\left\{\begin{array}{l}
\Psi\left(x^{*}\right)=2 C_{1} \sinh \left(l x^{*}\right), \\
\Sigma\left(x^{*}\right)=2 C_{2} \frac{\sinh \left(l\left(x^{*}-1\right)\right)}{\cosh (l)-\sinh (l)},
\end{array}\right.
$$

where

$$
C_{1}=\frac{\Phi}{2 l} \frac{1-R_{1}}{\sinh \left(l S_{0}^{*}\right)}\left\{\frac{\varphi \sigma+R_{3} l \operatorname{coth}\left(l S_{0}^{*}\right)}{R_{1} R_{2} \operatorname{coth}\left(l\left(S_{0}^{*}-1\right)\right)-\operatorname{coth}\left(l S_{0}^{*}\right)}+R_{3} l\right\},
$$




$$
C_{2}=\frac{\Phi}{2 l} \frac{\left(1-R_{1}\right)\left\{\varphi \sigma+l R_{3} \operatorname{coth}\left(l S_{0}^{*}\right)\right\}\{\cosh (l)-\sinh (l)\}}{\left\{R_{1} R_{2} \cosh \left(l\left(S_{0}^{*}-1\right)\right)-\operatorname{coth}\left(l S_{0}^{*}\right) \sinh \left(l\left(S_{0}^{*}-1\right)\right)\right\}}
$$

The eigenvalue problem for the temperature profiles in both phases (liquid and vapour) is

$$
\begin{aligned}
& \left(\frac{d^{2}}{d x^{* 2}}-E_{l i q} \sigma-l^{2}\right) \phi_{l i q}+\frac{1}{S_{0}^{*}} \frac{d \Psi}{d x^{*}}=0, \\
& \phi_{l i q}(0)=0, \phi_{l i q}\left(S_{0}^{*}\right)=-\frac{\Phi}{S_{0}^{*}}, \\
& \left(\frac{d^{2}}{d x^{* 2}}-\frac{E_{\text {vap }} \kappa R_{1}}{C} \sigma-l^{2}\right) \phi_{v a p}+\frac{1}{S_{0}^{*}-1} \frac{R_{1} R_{2} \kappa}{C} \frac{d \Sigma}{d x^{*}}=0, \\
& \phi_{\text {vap }}(1)=0, \phi_{\text {vap }}\left(S_{0}^{*}\right)=\frac{\Phi}{1-S_{0}^{*}} .
\end{aligned}
$$

The solution of (15) for the eigenfunctions of the temperature profiles is

$$
\begin{aligned}
\phi_{l i q}\left(x^{*}\right)= & \frac{C_{1}}{S_{0}^{*}} \frac{2 l}{E_{l i q} \sigma}\left[\frac{\sinh \left(\gamma_{1} x^{*}\right)}{\sinh \left(\gamma_{1} S_{0}^{*}\right)}\left\{\cosh \left(\gamma_{1} S_{0}^{*}\right)-\cosh \left(l S_{0}^{*}\right)\right\}\right. \\
& \left.+\left\{\cosh \left(l x^{*}\right)-\cosh \left(\gamma_{1} x^{*}\right)\right\}\right]-\frac{\Phi}{S_{0}^{*}} \frac{\sinh \left(\gamma_{1} x^{*}\right)}{\sinh \left(\gamma_{1} S_{0}^{*}\right)}, \\
\phi_{\text {vap }}\left(x^{*}\right)= & \frac{2 l R_{2} C_{2}}{\left(S_{0}^{*}-1\right) E_{\text {vap }} \sigma\{\cosh (l)-\sinh (l)\}}\left\{\frac{\sinh \left(\gamma_{2}\left(x^{*}-1\right)\right)}{\sinh \left(\gamma_{2}\left(S_{0}^{*}-1\right)\right)}\right. \\
& \left\{\cosh \left(\gamma_{2}\left(S_{0}^{*}-1\right)\right)-\cosh \left(l\left(S_{0}^{*}-1\right)\right)\right\} \\
& \left.+\cosh \left(l\left(x^{*}-1\right)\right)-\cosh \left(\gamma_{2}\left(x^{*}-1\right)\right)\right\} \\
& -\frac{\Phi}{\left(S_{0}^{*}-1\right)} \frac{\sinh \left(\gamma_{2}\left(x^{*}-1\right)\right)}{\sinh \left(\gamma_{2}\left(S_{0}^{*}-1\right)\right)}
\end{aligned}
$$

where $\gamma_{1}=\sqrt{l^{2}+E_{l i q} \sigma}$ and $\gamma_{2}=\sqrt{l^{2}+\frac{E_{v a p} \kappa R_{1}}{C} \sigma}$.

The relationship between the growth rate and wave number can be obtained using the energy balance across the liquid-vapour interface. This relationship is obtained by substituting (12) into the first order of the energy jump condition at the phase-change front, which yields

$$
\varphi H_{l i q} \sigma \Phi=\left[\left\{\frac{d \phi_{l i q}}{d x^{*}}+\frac{\Theta_{0}}{\kappa} \frac{d \phi_{\text {vap }}}{d x^{*}}\right\}-H_{l i q} \frac{d \Psi}{d x^{*}}\right]_{x^{*}=S_{0}^{*}} .
$$


The growth rate $\sigma$ in equation (18) has multiple solutions because $\gamma_{1}$ and $\gamma_{2}$ depend on $\sigma$; this non-linear equation must be solved numerically for $\sigma$. For this, Maple's implicitplot routine has been used. In this case, this method is adequate, but in some related problems [15] matters are not so simple.

\section{Transition to instability}

In this section, the possible types of transition to instability and the effect of the critical modified Rayleigh number on the stability of the front for short, medium and long wavelength disturbances will be discussed. Before doing so, however, it is important to find out the critical modified Rayleigh numbers for both zero wave number and infinite wave numbers, which play important roles in the changeover of the stable system to unstable.

\subsection{Long wavelength disturbances}

From the dispersion Eq. (18), three different special cases are derived. The key variation in these cases is the assumption of different modes of heat transport (see Table 1), where 'diff' and 'adv' stand for diffusion and advection, respectively.

\begin{tabular}{|c|c|c|}
\hline Boundary conditions & Basic state & Perturbed state \\
\hline \hline & & Only diff in the entire system \\
& & $\left(d \Psi / d x^{*}\right.$ and $d \Sigma / d x^{*}$ equal to zero in (15)) \\
Isothermal temperature & no & \\
at the boundaries & through & Adv \& diff in the liquid phase \\
with fixed pressure & flow & $\left(d \Sigma / d x^{*}\right.$ equal to zero in (15)) \\
& & Adv \& diff in the vapour phase \\
& & $\left(d \Psi / d x^{*}\right.$ equal to zero in (15)) \\
& & Adv \& diff in the entire system (18) \\
\hline
\end{tabular}

Table 1: List of problems under consideration

The asymptotic conditions on critical modified Rayleigh numbers for long wavelengths for the above different cases are given in Table 2. The procedure followed to obtained these critical modified Rayleigh numbers has been discussed in [14].

The above different critical modified Rayleigh numbers for long wavelengths based on the different modes of heat transfer, are plotted in Figure 2 as function of the ratio of the temperature contrasts across the liquid and vapour 
Table 2: List of critical modified Rayleigh numbers for long wavelength

\begin{tabular}{|c|c|}
\hline Critical modified Rayleigh number, $\left|R_{30}^{\text {crit }}\right|$ & Modes of heat transport \\
\hline \hline$\frac{\left(\kappa R_{1} R_{2}+\Theta_{0}\right)}{\Theta_{0} H_{l i q} R_{1} R_{2}\left(R_{1}-1\right)}\left(\frac{\kappa+\Theta_{0}}{\kappa}\right)^{2}$ & Only diff in the entire system \\
\hline$\frac{2\left(\kappa R_{1} R_{2}+\Theta_{0}\right)}{\Theta_{0} R_{1} R_{2}\left(1+2 H_{l i q}\right)\left(R_{1}-1\right)}\left(\frac{\kappa+\Theta_{0}}{\kappa}\right)^{2}$ & Adv \& diff in the liquid phase \\
\hline$\frac{2 C\left(\kappa R_{1} R_{2}+\Theta_{0}\right)}{\Theta_{0} R_{1} R_{2}\left(\Theta_{0}+2 C H_{l i q}\right)\left(R_{1}-1\right)}\left(\frac{\kappa+\Theta_{0}}{\kappa}\right)^{2}$ & Adv \& diff in the vapour phase \\
\hline$\frac{2 C\left(\kappa R_{1} R_{2}+\Theta_{0}\right)}{\Theta_{0} R_{1} R_{2}\left(R_{1}-1\right)\left(\Theta_{0}+\left(2 H_{l i q}+1\right) C\right)}\left(\frac{\kappa+\Theta_{0}}{\kappa}\right)^{2}$ & Adv \& diff in the entire system \\
\hline \hline
\end{tabular}

layers $\Theta_{0}$ and of the density ratio $R_{1}$. Figure 2 (a) shows that when $\Theta_{0}$ is very large or very small the system is more stable than for intermediate values; this is because these limits correspond to a strong thermal gradient on one side or the other of the front, which has a stabilising effect through vertical diffusion. Furthermore, a system which is governed by both advection and conduction is more unstable for zero wave number then a purely conductive system. Figure 2 (b) surprisingly shows that the interface becomes more stable as $R_{1} \rightarrow 0$; because the Rayleigh-Taylor mechanism is not effective for $l=0$, and hence our physical intuition is misleading in this case. 


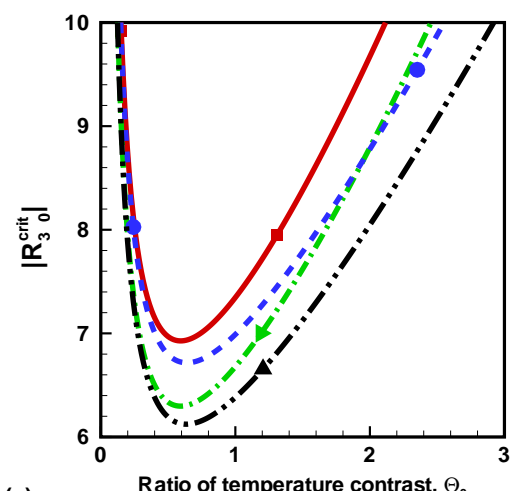

(a) Ratio of temperature contrast, $\Theta_{0}$

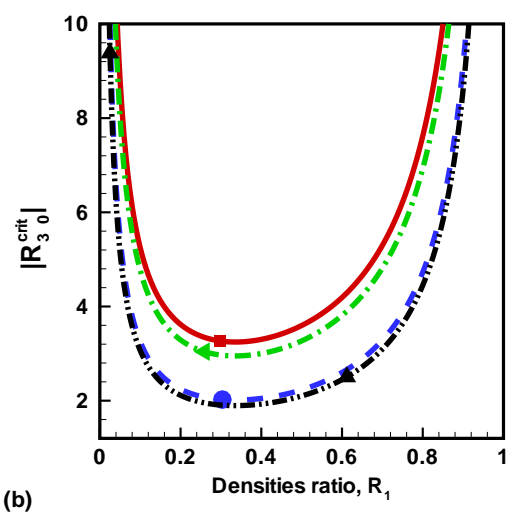

(b)

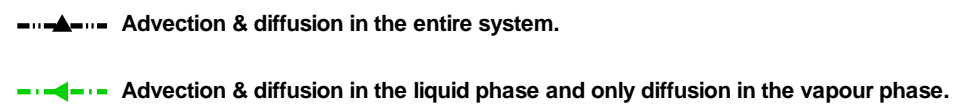

- Only diffusion in the liquid and advection \& diffusion in the vapour phase.

Only diffusion in the entire system.

Figure 2: The critical modified Rayleigh number for long wavelength verses (a) ratio of the temperature contrast and (b) ratio of the densities $R_{1}$, where $R_{2}=8.75, C=1.96, \kappa=$ $4, H_{l i q}=5$.

\subsection{Short wavelength disturbances}

We will use asymptotic analysis to help us locate the stability boundary in parameter space. We will focus in particular on the critical modified Rayleigh number for infinite wave number. For this let $\sigma=\sigma^{*} l$ and $\sigma^{*}=\sigma_{0}+\frac{\sigma_{1}}{l}+O\left(\frac{1}{l^{2}}\right)$ and take $l \rightarrow \infty$; then expanding (18) in asymptotic series in $l$ and equating the terms proportional to $l$, gives

$$
\sigma_{0} \sim \underbrace{\frac{R_{1} R_{2}+1}{\varphi H_{l i q} R_{1}\left(R_{2}+1\right)}\left\{-\frac{1}{S_{0}^{*}}-\frac{\Theta_{0}}{\kappa} \frac{1}{1-S_{0}^{*}}\right\}}_{\text {first term }}-\underbrace{\frac{1-R_{1}}{R_{2}+1} \frac{R_{2} R_{3}}{\varphi}}_{\text {second term }} .
$$

From (19) it is clear that the first term has always negative sign because the dimensional parameters in the first term are all positive real numbers. So the first term, which represents the diffusive heat transport process, has a stabilising effect on the liquid-vapour phase change front.

Now here we have two different cases to discuss. If the lighter fluid is above the heavier fluid, i.e., $R_{3}>0$, then the second term has a negative sign (recall that $R_{1}<1$ ) and the front is stable for infinite wave numbers.

Alternatively, if $R_{3}<0$, which means that heaver fluid (liquid) is above the 
lighter fluid (vapour), then the second term in (19) has a positive sign and has a destabilising effect on the liquid-vapour phase change front. It is the competition between the first and second terms which will determine the nature of the liquid-vapour phase change front. Now we will find the critical modified Rayleigh number for infinite wave numbers. For this, in the case of marginal stability $\sigma_{0}=0$, and solving (19) for $R_{3}$ and using the value of $S_{0}^{*}$ from (11), we have

$$
R_{3}^{c r i t} \sim R_{3, \infty}^{\text {crit }}=\frac{2\left(R_{1} R_{2}+1\right)}{H_{l i q} R_{1} R_{2}\left(R_{1}-1\right)} \frac{\kappa+\Theta_{0}}{\kappa}, \quad \text { as } \quad l \rightarrow \infty .
$$

This tells us immediately that the instability can only occur for sufficiently large, negative values of the modified Rayleigh number $R_{3}$. The condition on critical modified Rayleigh number for infinite wave numbers for the three different cases (see Table 2) is the same as (20), because the horizontal diffusion dominates advection for short waves.

\subsection{Anomaly of spontaneous transition}

In this section, we will explore the possibility of spontaneous transitions to instability depending upon the position of the liquid-vapour phase change front $S_{0}^{*}$. Figure 3 displays the neutral stability curves $(\sigma=0)$ for the different heat transport processes and front positions (see Table 1). The critical modified Rayleigh number for the onset, $\left|R_{3}^{\text {crit }}\right|$, varies with front position $S_{0}^{*}$ and wavelength $l$. The lowest critical modified Rayleigh numbers in figure 3 (a) and (c) are found by searching for the minimum value of $\left|R_{3}^{\text {crit }}\right|$ for all wavelengths. Figure 3 (a) shows that the system with only diffusion has no minimum value of $\left|R_{3}^{\text {crit }}\right|$. The liquid-vapour interface become unstable first for short-waves and then for long-waves. For the case when the liquid-vapour interface is equidistant from the liquid and vapour boundaries, the transition to instability is spontaneous (see figure 3 (b)). Figure 3 (c) shows that the lowest critical modified Rayleigh number of the first unstable mode, $\left|R_{3 \text {,mini }}^{\text {crit }}\right|$, is 6.26 . This occurs for the system with both advection and diffusion for the critical wavelength $l^{\text {crit }}=2.78$. These results are for the particular set of parameters chosen, thus the critical values of $\left|R_{3}^{\text {crit }}\right|$ cannot be assumed to be universal.

The overall feature of these special cases is that the advective heat transport is not essential for instability, but it encourages the unstable behaviour. The same observations were made by [14] while investigating the same system with mixed boundary conditions (cooling flux at the liquid boundary). 


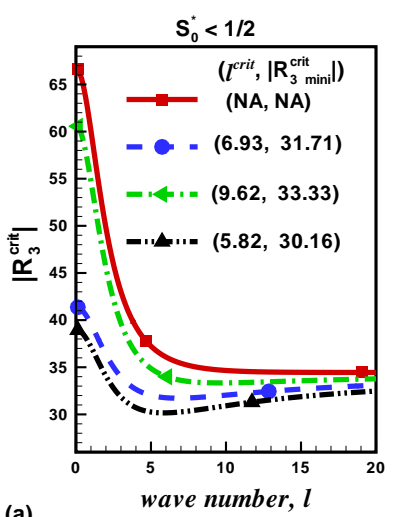

Figure 3: The dependence of $\left|R_{3}^{\text {crit }}\right|$ on the wave number $l$ for the different modes of heat transport. For different curves associated with different heat transport processes see Fig. 2 .

\subsubsection{Liquid-vapour interface is near to the vapour (lower) boundary}

The imposition of interface to be near the vapour (lower) boundary, i.e., $S_{0}^{*}>\frac{1}{2}$, represents the configuration of a liquid dominated geothermal system. The representative example of how the growth rate $(\sigma)$ varies with the wave number $(l)$ for the different transport processes, $\left|R_{3}\right|$ is the curve parameter, is illustrated in Figure 4. The short and long wavelength disturbances are found to be stable since $\sigma<0$. Short wavelength disturbances (large $l$ ) are stabilised by horizontal thermal diffusion which eliminates the variations in the horizontal direction of the perturbed front, whereas long wavelength disturbances (small $l$ ) are stabilised by vertical diffusion. Medium wavelength disturbances become unstable as the modified Rayleigh number increases: this is a reflection of the buoyant instability, because the denser fluid (liquid) is above the less dense fluid (vapour).
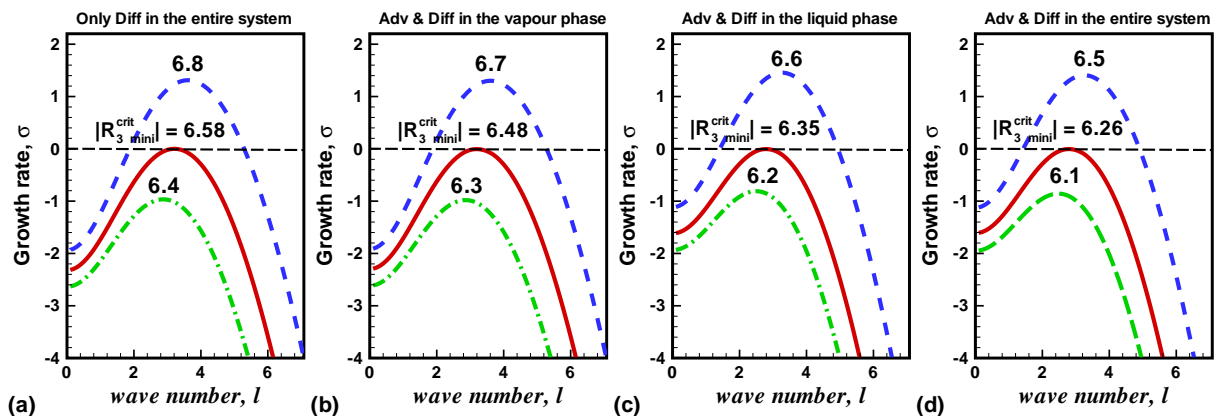

Figure 4: Medium wavelength disturbances of the system when the liquid-vapour interface is near to the vapour (lower) boundary, i.e., $S_{0}^{*}>\frac{1}{2}$. 


\subsubsection{Liquid-vapour interface is equidistant from the boundaries}

Let us assume that the position of the liquid-vapour phase change front is the middle of the porous layer, i.e., $S_{0}^{*}=\frac{1}{2}$, so (11) yields

$$
k=\Theta_{0} .
$$

Substituting (21) into the necessary conditions on the modified Rayleigh numbers for the short and long wavelengths for the pure conductive heat transfer in the entire system case, gives

$$
R_{3,0}^{\text {crit }}=\frac{4\left(R_{1} R_{2}+1\right)}{H_{\text {liq }} R_{1} R_{2}\left(R_{1}-1\right)}=R_{3, \infty}^{\text {crit }}
$$

which shows that if $\left|R_{3}\right|=\left|R_{30}^{\text {crit }}\right|=\left|R_{3 \infty}^{\text {crit }}\right|$ (recall $R_{3}<0$, liquid above vapour), then $\sigma(l)=0$. The instability takes place spontaneously, in the sense that as $\left|R_{3}\right|$ is increased, the system becomes unstable, i.e. $\sigma(l)=0$, for all wave numbers $l$ at once (see figure $5(\mathrm{a})$ ). The same type of transition to instability was found by Il'ichev \& Tsypkin [11], while considering that the phase change temperature depends on pressure but we take the temperature at the interface as a constant. Furthermore, if $\left|R_{3}\right|>\left|R_{3}^{\text {crit }}\right|$ then the system is unstable, at fixed values of the pressure and temperature on the upper and lower boundaries.

However, as we can see from Figures 5 (b) and (c), when any advection is included the spontaneous transition no longer occurs. This indicates that the interesting behaviour in Il'ichev \& Tsypkin [11] model is an artefact of taking a very simple model which neglected thermal advection.
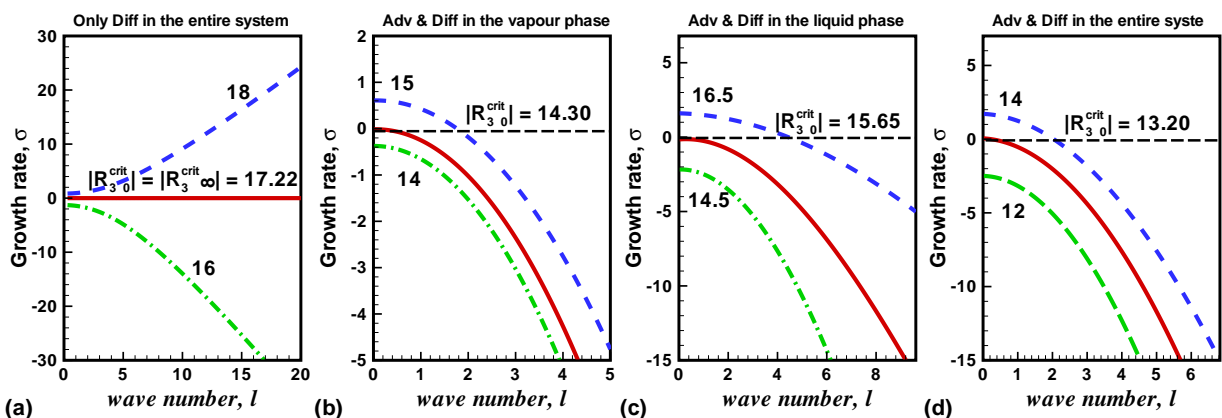

Figure 5: Transition to instability of the system when the liquid-vapour interface is equidistant from the liquid and vapour boundaries, i.e., $S_{0}^{*}=\frac{1}{2}$.

\subsubsection{Liquid-vapour interface is near to the liquid (upper) boundary}

The imposition of interface to be near the liquid (upper) boundary, i.e., $S_{0}^{*}<\frac{1}{2}$, represents the configuration of a vapour dominated geothermal 
system. The stability analysis reveals that the system with only diffusion is stable to long wavelength disturbances, whereas the short and medium wavelength disturbances are unstable for $\left|R_{3}\right| \geq\left|R_{3}^{\text {crit }}\right|$ (see Figure 6 (a)). This short-wave instability shows that the horizontal thermal diffusion becomes unimportant. However, when any advection is included, the liquid-vapour interface becomes unstable first to medium wavelength disturbances as the modified Rayleigh number increases, through a Rayleigh-Taylor instability.

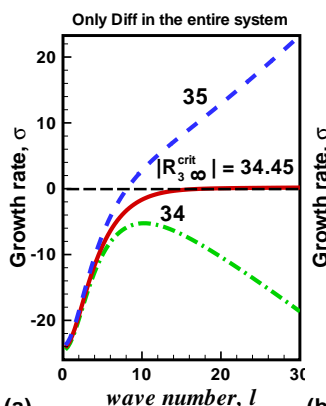

(a)

Figure 6: Transition to instability of the system when the liquid-vapour interface is near to the liquid (upper) boundary, i.e., $S_{0}^{*}<\frac{1}{2}$.

\section{Discussion and conclusions}

This study has investigated the two-dimensional stability of the liquidvapour interface in a fluid saturated porous layer with fixed temperatures at the top and bottom boundaries. The base state is one dimensional without through flow and the perturbation is considered both with and without thermal advection.

In the liquid- and vapour-dominated regimes, there is a Rayleigh-Taylor instability which is damped by horizontal diffusion but in which the instability mechanism never precisely balances the stabilising effects for all wavenumbers.

For pure diffusive heat transport process in the entire system with equal distribution of the two phases, the transition to instability is spontaneous, which is completely in agreement with the results shown by Il'ichev \& Tsypkin [11]. However, the inclusion of advection means that spontaneous transition is no logner possible. The model with a pressure-independent phasechange temperature shares with Il'ichev \& Tsypkin's model [11] the property that it exhibits a spontaneous transition when $S_{0}=1 / 2$ and advection is 
neglected, and that this strongly suggests that the explanation for this transition lies in the transport mechanisms rather than the pressure/temperature relationship.

A further surprising result is that when the densities ratio $R \rightarrow 0$, the liquid-vapour interface becomes more stable because the Rayleigh-Taylor mechanism loses effectiveness for long wavelength disturbances.

Acknowledgement. The author wish to express his very sincere thanks to the reviewers for their valuable comments and suggestions on this work.

\section{Nomenclature}

\section{Latin}

$c_{p}$ specific heat

$g$ acceleration due to gravity

$H$ reciprocal of Stefan number

$K$ permeability

$k$ thermal conductivity

$L$ thickness of the low permeable layer

$l$ wave number

$P$ pressure

$q$ heat flux per unit area

$S$ location parameter of the interface

$T$ temperature

$t$ time

$x$ vertical coordinate

$y$ horizontal coordinate

\section{Greek symbols}

$\epsilon$ perturbation parameter

$\kappa$ thermal conductivities ratio

$\lambda$ latent heat

$\mu$ dynamic viscosity

$\nu$ kinematic viscosity

$\rho$ density

$\sigma$ spectral parameter

$\sigma^{*}$ asymptotic spectral parameter

$\Theta$ dimensionless temperature

$v$ fluid flow velocity

$\varphi$ porosity

\section{Dimensionless quantities}

$C$ specific heat ratio

$E$ heat capacity ratio

$R$ kinematic viscosity ratio

$R_{1}$ density ratio 
$R_{2}$ dynamic viscosity ratio

$R_{3}$ modified Rayleigh number

\section{Subscripts}

$L$ liquid boundary

liq liquid phase

$m$ porous medium

ref reference quantity

$S$ at the phase transition front

$s$ porous skeleton
$V$ vapour boundary

vap vapour phase

0 base state

1 perturbed state

\section{Superscripts}

mini minimum

* dimensionless quantity

0 base state

1 perturbed state

\section{References}

[1] L. Rayleigh, Investigation of the character of the equilibrium of an incompressible heavy fluid of variable density, Proceedings of the London Mathematical Society 14 (1883) $170-177$.

[2] G. Bodvarsson, K. Pruess, M. Lippmann, Modeling of geothermal systems, Journal of Petroleum Technology 38 (9) (1986) 1007-1021.

[3] A. H. Truesdell, D. E. White, Production of super-heated steam from vapor-dominated geothermal reservoirs, Geothermics 2 (1973) 154 173.

[4] S. E. Ingebritsen, M. L. Sorey, Vapor-dominated zones within hydrothermal systems: Evolution and natural state, Journal of Geophysical Research 93 (1988) 13,635 - 13,655.

[5] R. M. Young, Phase transitions in one-dimensional steady state hydrothermal flows, Journal of Geophysical Research 101 (1996) 18,011 18,022 .

[6] A. Il'ichev, G. Tsypkin, Catastrophic transition to instability of evaporation front in a porous medium, European Journal of Mechanics B/Fluids 27 (2008) $665-677$. 
[7] G. Tsypkin, A. Il'ichev, Gravitational stability of the interface in water over steam geothermal reservoirs, Transport in Porous Media 55 (2004) $183-199$.

[8] J. M. Straus, G. Schubert, One-dimensional model of vapor-dominated geothermal systems, Journal of Geophysical Research 86 (1981) 9433 9438 .

[9] A. Il'ichev, G. Tsypkin, Influence of advective transfer of energy on stability of water over steam in geothermal systems, Doklady Physics 56 (2011) 227-231.

[10] Z. H. Khan, D. Pritchard, Liquid-vapour fronts in porous media: Multiplicity and stability of front positions, International Journal of Heat and Mass Transfer 61 (0) (2013) $1-17$.

[11] A. T. Il'ichev, G. G. Tsypkin, Transition to instability of the interface in geothermal systems, European Journal of Mechanics B/Fluids 24 (2005) $491-501$.

[12] A. Il'ichev, G. Tsypkin, Instabilities of uniform filtration flows with phase transition, Journal of Experimental and Theoretical Physics 107 (2008) $699-711$.

[13] B. Sarler, Stefan's work on solid-liquid phase changes, Engineering Analysis with Boundary Elements 16 (1995) $83-92$.

[14] Z. H. Khan, Transition to instability of liquidvapour front in a porous medium cooled from above, International Journal of Heat and Mass Transfer 70 (0) (2014) $610-620$.

[15] Z. H. Khan, Modelling moving evaporation fronts in porous media, $\mathrm{PhD}$ thesis, University of Strathclyde, Glasgow, Scotland (2012).

[16] P. G. Drazin, Introduction to Hydrodynamic Stability, Cambridge University Press, Cambridge, 2002. 